\title{
Animal models of Parkinson's disease and L-dopa induced dyskinesia: How close are we to the clinic?
}

\author{
Emma Lane $\cdot$ Stephen Dunnett
}

Received: 1 March 2007 / Accepted: 27 August 2007 / Published online: 25 September 2007

(C) Springer-Verlag 2007

\begin{abstract}
Background Several different animal models are currently used to research the neurodegenerative movement disorder Parkinson's disease (PD).

Results Models based on the genetic deficits associated with a small percentage of sufferers demonstrate the pathological accumulation of $\alpha$-synuclein characteristic of the disease but have few motor deficits and little neurodegeneration. Conversely, toxin-based models recreate the selective nigrostriatal cell death and show extensive motor dysfunction. However, these toxin models do not reproduce the extra-nigral degeneration that also occurs as part of the disease and lack the pathological hallmark of Lewy body inclusions.

Discussion Recently, several therapies that appeared promising in the MPTP-treated non-human primate and 6-OHDAlesioned rat models have entered clinical trials, with disappointing results. We review the animal models in question and highlight the features that are discordant with PD, discussing if our search for pharmacological treatments beyond the dopamine system has surpassed the capacity of these models to adequately represent the disease.
\end{abstract}

Keywords Parkinson's disease .

L-dopa-induced dyskinesia · 6-OHDA lesioned rat .

MPTP-treated primate $\cdot$ Animal models

\section{Introduction}

The empirical pathway in the development of therapeutics for human disease states is to test them against the disease

E. Lane $(\bowtie) \cdot S$. Dunnett

School of Bioscience, Cardiff University,

Cardiff, UK

e-mail: LaneEL@cf.ac.uk concerned. However, the screening and initial testing of novel compounds directly in patients is generally deemed inappropriate; therefore, the development of animal models provides an alternate pathway that allows the development and refinement of therapeutics before clinical trials. An animal model is defined as an animal that has a disease or injury similar to the human condition. Ideally, it should have a similar aetiology and function to the human equivalent, and it is necessary that as many aspects of the disease are replicated as possible to avoid confounding and misleading results, which in turn may hinder the development of new therapeutic approaches. The accuracy with which the model reflects the disease state is, therefore, dependent on prior knowledge of the aetiology. As this knowledge develops within both clinical and pre-clinical settings, so must the disease models. The validation of the model as an accurate representation of the disease should also be influenced by clinical experience, and the success or failure of drug trials should feed back into our understanding not only of the disease but also of the limitations of the model used. The selection and validation of the model must also be appropriate to the mechanism of action of the proposed therapeutic target. We will have different requirements of a model to test a neuroprotective drug that alters the pathogenic course of disease, than to test whether another compound can alleviate particular classes of symptoms. In the first case, the model has to provide a causal mechanism that reflects the disease process, even if we focus on surrogate markers that bear little resemblance in the animals to that observed in patients; in the second case, the neural systems and behavioural phenotype seen in the animal will have to mimic closely the human symptoms, even if the causal method of inducing degeneration is quite different.

Parkinson's disease (PD) is a progressive neurodegenerative motor disorder characterised by the cardinal symptoms of resting tremor, bradykinesia, rigidity and postural 
instability. The most striking aspects of the pathology of PD are the loss of pigmented dopaminergic neurones located in the substantia nigra and the presence of eosinophilic inclusions, termed Lewy bodies, in surviving cells. The consequences are a motor disorder characterised by tremor, rigidity and bradykinesia. A predominantly sporadic disease, the likely cause of PD is currently considered to involve a combination of genetically determined vulnerability and exposure to toxins in the environment. In a small percentage of patients, the disease is familial, with a direct genetic defect responsible and with their identification the developing power of transgenic, knock-in and knockout technologies has been utilised in an approach to developing pathogenetically appropriate models. In the most detailed behavioural characterisation yet available, Fleming et al. (2005) have provided a comprehensive analysis of the behavioural characteristics of these mutant mice including spontaneous activity, gait, motor coordination, sensory responses, and sensorimotor impairments, that reproduce some features of the human disease. However they are not yet associated with the level of reliability, magnitude and progression that would provide an ideal baseline for assessing therapeutic responses (Fleming et al. 2005). Finally, several of these mutations have been associated with marked motor impairments in drosophila models (Feany and Bender 2000; Park et al. 2005), which are useful for high-throughput screening of genetic associations of the pathology, but are of less use for pharmaceutical development.

As these genetic models have not yet added significantly to pharmacotherapeutic development and are extensively reviewed elsewhere (Fleming et al. 2005), they will not be discussed further in this article. Instead we evaluate the traditional and more commonly used toxin-based models of PD used to evaluate potential therapeutic strategies, the 1methyl 4-phenyl 1,2,3,6-tetrahydropyridine (MPTP)-treated primate and the 6-hydroxydopamine (6-OHDA) lesioned rat. Whilst having a sound basis for their development and enabling major advances in the understanding of pathological mechanisms and in the development of therapies, they may reflect a relatively simple concept of a disease now known to be more complex.

\section{6-Hydroxydopamine}

The most commonly used toxin-based models, the 6OHDA-lesioned rat and the MPTP-treated non-human primate have been used extensively in the search for PD therapies. Previously, these animal models of PD have proven to have a relatively good predictive validity for antiparkinsonism drugs, although a model is only as good as the behavioural tests used to assess therapeutic benefit. The popularly used 6-OHDA lesioned rat was a step forward from the use of reserpine, one of the first rodent models of PD. Whilst the reserpine model was fundamental to the development of L-dopa (Carlsson et al. 1957), the monoamine depletions it evokes are transient and non-selective and there is no concomitant cell death. The 6-OHDA lesioned rat (or mouse) provides a model of permanent dopaminergic depletion selective to the nigrostriatal pathway, the area predominantly affected in PD. Methodologically, although 6-OHDA is a non-specific catecholaminergic toxin, selective nigrostriatal dopaminergic degeneration is achieved by stereotaxic surgical administration with noradrenaline transporter blockade. Bilateral nigrostriatal depletion produces a motor syndrome so severe that the animals are unable to feed or hydrate without assistance (Zigmond and Stricker 1972, 1973), therefore the toxin is commonly injected unilaterally into specific areas along the nigrostriatal pathway (Ungerstedt 1971a, b; Marshall et al. 1976). This gives a unilateral motor deficit that can be evaluated in a variety of different tests. A rat with a unilateral nigrostriatal lesion exhibits postural bias to the ipsilateral side, which is transformed into strong ipsilateral turning ('rotation') after activation by injection of a stimulant drug such as amphetamine. Extracellular dopamine is increased in the intact striatum creating an imbalance in motor activation resulting in the rotational response. The animals will also rotate ipsilaterally after a variety of activating stimuli, such as stressors (e.g. placing in a novel environment, placing on an ice cold surface or tailpinch), although the pharmacological methods of activation are the most widely used because of their reliability and reproducibility.

Drugs are often initially screened for anti-parkinsonian potential judged by their ability to induce contralateral rotational behaviour indicating restoration of function in the lesioned striatum, as opposed to increased dopaminergic function in the intact hemisphere. The dopamine precursor L-dopa produces robust contralateral rotations, as do dopamine agonists such as bromocriptine and apomorphine (Ungerstedt 1971a, b; Reavill et al. 1983). The contralateral direction of rotation suggests preferential activation of the outputs from the striatum on the lesioned side, which is thought to be dependent upon the development of postsynaptic dopamine receptor supersensitivity on the striatal target neurons after their dopaminergic deafferentation. Indeed, this behavioural data was the initial basis for the supersensitivity hypothesis (Ungerstedt 1971a, b), which has been subsequently confirmed on many occasions by changes in both the sensitivity and binding in receptor ligand assays, and supported by the fact that agonists such as apomorphine induce strong contralateral turning at very low doses that are sub-threshold for any detectable activating effect in normal animals (Ungerstedt 1971a, b).

Other tests are more useful for evaluating voluntary asymmetric motor behaviours in the undrugged animal. For 
example, unilateral lesions induce an asymmetry in the use of the forelimb (cylinder, stepping, paw reaching) and neglect of contralateral stimuli (neurological sensorimotor tests, placing, whisker tests, corridor test; Olsson et al. 1995; Schwarting and Huston 1996; Whishaw et al. 1997; Dowd et al. 2005; Monville et al. 2005). L-dopa is able to ameliorate several of these deficits but is complicated by the development of competing stereotypical and dyskinetic behaviours associated with enhanced receptor sensitivity. Thus, for example, Ljungberg and Ungerstedt (1976) first reported that L-dopa can alleviate eating deficits in the first few days after bilateral lesions, but within a week, the development of supersensitivity induced stereotyped gnawing that blocked the animal's ability to engage in voluntary drinking (Ljungberg and Ungerstedt 1976). More recently, we have found that the most sensitive test of the therapeutic effect of L-dopa is the placing test of forelimb contact placing triggered by whisker stimulation as the animal is lowered towards the corner of the bench (Monville et al. 2005). In this test, the animal is held closely, allowing free movement of the single paw placement response whilst restraining the orientation of the head and other three paws, and at the same time eliminating interference from competitive stereotypies.

\section{1-Methyl-4-phenyl-1,2,3,6-tetrahydropyridine}

MPTP is a highly lipophilic substance that readily crosses the blood brain barrier where it is metabolised to $\mathrm{MPP}^{-}$by non-neural cells. $\mathrm{MPP}^{-}$can then be taken up into neurons through the dopamine transporter, where it inhibits complex I of the mitochondrial respiratory chain (Nicklas et al. 1985). This produces an extensive set of behavioural deficits that are strikingly similar to the clinical parkinsonian syndrome (Jenner et al. 1984; Langston et al. 1984) displaying bradykinesia, rigidity and postural abnormalities, and its discovery revolutionised research into PD pharmacotherapy. Variations of clinical PD rating scales are used to assess the severity of the motor disability, and often this is concurrent with measures of spontaneous locomotor activity, which becomes severely reduced after MPTP. Until recently, the high face validity of the MPTP-treated nonhuman primate model has led to a high predictive validity in determining therapeutics that are effective in ameliorating symptoms of the disease although this has proven emphatically less so in the evaluation of neuroprotective and disease modifying strategies. L-dopa produces significant increases in motor performance with large reduction in disability. Similarly, dopamine agonists and Catechol Omethyltransferase inhibitors, all now available in the clinic, produce improved parkinsonian disability (Grondin et al. 1996; Smith et al. 1997; Maratos et al. 2001).
Behaviourally, the primate model remains the best use of MPTP but the toxin has been used in a variety of other species including zebra fish, dogs and mice. Although rats, rabbits and guinea pigs are relatively insensitive to MPTP (Donnan et al. 1986), it has seen widespread use in the mouse especially in studies of neurochemistry and neuropathology and in evaluating strategies for neuroprotection. The MPTP-treated mouse does not always show clinically related parkinsonian signs but can produce stable dopaminergic nigrostriatal deficits. However, it has become apparent that the susceptibility to degeneration is dependent on the age, gender and strain of mouse used, and the variability with which different strains respond to MPTP must be highlighted and strict protocols should be adhered to (Jackson-Lewis and Przedborski 2007).

\section{Validity of animal models for assessing symptomatic therapies}

Several pharmacotherapies have looked highly promising in these pre-clinical animal models, producing impressive improvements in motor function. However, this success has not followed through, and in phases I and II, clinic trials are demonstrating little, if any, efficacy. NS2330, BTS 74398 and brasofensine are all non-selective monoamine uptake inhibitors with almost equal potency at the noradrenaline, dopamine and serotonin uptake transporters. In MPTP-treated primates, such drugs not only exhibit the ability to reduce motor deficits and increase locomotion but also appear to restore normal motor behaviours such as grooming, without the driven stereotypical behaviours that can be seen after Ldopa (Hansard et al. 2004). Moreover, they induce ipsilateral rotations in 6-OHDA lesioned rats that do not sensitise with long-term administration suggesting that they have a very low dyskinesiogenic potential (Lane et al. 2005). Additionally, in MPTP primates that have been 'primed' with chronic L-dopa to display dyskinesia, the administration of BTS 74 398 failed to induce dyskinesia whilst still producing behavioural improvement further supporting the potential benefit of these compounds both in early stage unmedicated patients and in later stage dyskinetic patients (Hansard et al. 2004). Disappointingly, when each of these has been taken into the clinic, there has been a very limited level of functional improvement and a higher incidence of side effects (Frackiewicz et al. 2002; Bara-Jimenez et al. 2004). Similarly, partial dopamine $\mathrm{D}_{2}$ agonists, which, having demonstrated antiparkinsonian and anti-dyskinetic properties in MPTP-treated monkeys, produced varied clinical outcomes including worsening both PD symptoms and dyskinesia (Baronti et al. 1992). This pattern has been repeated with other potential anti-parkinsonian drugs such as the adenosine $\mathrm{A}_{2 \mathrm{~A}}$ antagonists, adrenergic $\alpha_{2 \mathrm{~A}}$ antagonists and 5- $\mathrm{HT}_{1 \mathrm{~A}}$ agonists (for 
reviews see Linazasoro 2004 and Fox et al. 2006). Problems with bioavailability, tolerability and lack of specificity have been variously cited as reasons for these failures, but we must also consider whether our current development of therapeutics has surpassed the capacity of these popularly used models to characterise the disease adequately.

So what are the discordant features of the models that could be giving us false leads in the development of pharmacotherapeutics? The first and possibly highly significant deviation from the disease state with both toxin models is the temporal profile of cell death. The neurodegeneration in $\mathrm{PD}$ is a progressive (and presumably ongoing, active) process producing a slow, continual deterioration over a long period of time. Patients go from mild movement abnormalities, which are first observed with about $60 \%$ loss of dopaminergic innervation of the striatum through severe disease states, which correlate with the progressive death of the dopaminergic nigrostriatal neurones (Bernheimer et al. 1973). In contrast, acute insults in both toxin models produce severe dopaminergic depletions that are, in general, greater than $90 \%$ complete over a relatively short period of time. Once the cell death is complete, within 2 weeks of toxin administration in the case of 6-OHDA, a few days in nonhuman primates treated with MPTP, the degeneration is stable with no progression. This produces animals with a severe and static disease state that represents a snapshot of the later stage of the illness, but fails to incorporate its progressive nature or, consequently, the dynamic process of compensation. Levels of the toxin and administration regimes can be altered to produce a more gradual (Meissner et al. 2003) or more restricted (Kirik et al. 1998; Iravani et al. 2005) degeneration but generally they remain stable in contrast to the dynamic disease process.

The severity and selectivity of the damage produced by these toxins, which has historically been viewed as having high face validity with late stage PD may now be considered a less desirable feature. Specifically, 6-OHDA is typically injected unilaterally into the striatum, median forebrain bundle or substantia nigra of rats to eliminate, whether completely or partially, the nigrostriatal pathway. The extent of the damage is controlled by the quantity of toxin injected and the number and location of the injection sites, but typically the damage is severe and highly selective to the dopamine system. The peripheral injection of MPTP over several days produces a bilateral dopaminergic cell death predominantly affecting the A9 dopaminergic neurons of the substantia nigra (Jenner et al. 1984; Langston et al. 1984). Whilst the dopaminergic neurons of the ventral mesencephalon are the earliest and most extensive focus of pathology and cell death in PD patients, it is not the only neuronal system that is affected. Post-mortem analyses confirm moderate reductions in the amount of striatal serotonin with a concordant loss of serotonergic and also noradrenergic neurons from the dorsal raphé and locus coeruleus, respectively (Fahn et al. 1971). It has further been proposed that the pathology, as evidence by the presence of Lewy bodies, may actually occur in the locus coeruleus and dorsal raphé before the substantia nigra (Braak et al. 2002) although this is disputed (D. Brooks, personal communication). As, generally, newer drugs are not acting solely on the dopaminergic system, the extension of our models to include the degeneration in the important non-dopaminergic areas may be increasingly important. 6-OHDA can be taken up through both the dopamine and noradrenaline re-uptake transporters and is, therefore, potentially toxic to all catecholaminergic neurons. Moreover, if required, related toxins 5,6- and 5,7dihydroxytryptamine can be used to disrupt serotonin systems more or less selectively with catecholamine damage (Baumgarten et al. 1978). There is evidence for both hyperand hypo-serotonergic innervation of the striatum after 6OHDA lesions, and it may be dependent on the protocol used and the developmental stage of the animal at the time of lesion (Iwamoto et al. 1976; Breese et al. 1984; Zhou et al. 1991; Maeda et al. 2003). The reports on monoaminergic loss after MPTP administration are mixed. In both mice and primates, some studies suggest that the degeneration is predominantly dopaminergic with limited in other cell populations, whilst others report extensive serotonergic and noradrenergic loss (Pifl et al. 1991; Alexander et al. 1992). It may depend on the administration regime used, although it is clear that the loss of non-dopaminergic neurons is variable.

A defining pathological hallmark of PD is the presence of neuronal Lewy body inclusions throughout the nervous system, (indeed Lewy's original description focused on 'paralysis agitans' pathology in the nucleus basalis of Meynert Lewy 1914). A major drawback of the toxin-based models is the lack of similar proteinous aggregations. No such formations are observed after 6-OHDA lesioning, and whilst the accumulation of $\alpha$-synuclein has been reported in aged MPTP-treated primates this is not in the form of Lewy bodies (Forno et al. 1986; Kowall et al. 2000). Under certain treatment conditions MPTP-treated mice reportedly develop inclusions, which may be important in its use in investigating disease-modifying approaches but as stated above this model does not have the clinical deficits required to develop symptomatic treatments. The role of protein aggregations in the treatment of PD has not been fully determined but altered protein handling in the cell could impact on both receptor and transporter functions.

PD is a disease associated with aging that, whilst it may be predominantly affecting one side of the body more than the other, is generally a bilateral disorder. Unfortunately, due to the cost and practicalities of housing and maintaining animals into middle or old age, most studies are carried out in young animals, and this applies to both rodent and primate studies. Moreover, the unilateral lesion models are 
generally favoured because they allow the animals to maintain good health whilst exhibiting clear motor deficits on the contralateral side. After bilateral lesions, if the animals are severely depleted, they have severe problems regulating food and water intake, with attendant welfare concerns (Zigmond and Stricker 1972, 1973). Conversely, if the lesions are less complete, the animals rapidly recover from motor impairments making it difficult to evaluate the long-term effects of any experimental treatment. This means that what may be clinically relevant characteristic factors in the construct of these models are commonly overlooked. Furthermore, because specific genetic mutations have been identified, which contribute to risk associated with cases of familial $\mathrm{PD}$, it has been proposed that certain genetic combinations may contribute to an environmental susceptibility. The influence of these genetic factors cannot be easily determined in the rat or primate models, in particular when the pathogenic mechanism is presumed to be quite different in animals from that resulting in idiopathic disease in man. Changes have been observed in susceptibility to 6-OHDA or MPTP toxicity in mutant mice (see above), in whom genetic manipulations are easier, but the testing paradigms to demonstrate functional improvement are less well-developed and acute MPTP-treatment in the mouse only produces a temporary loss of dopamine in contrast to the permanent cell death in the non-human primate and in PD (Eidelberg et al. 1986).

Another key feature of the motor disorder is also missing from current animal models. One of the cardinal symptoms of PD is tremor, yet few animal models reproduce this type of motor dysfunction. Whilst there have been reports of tremor observed in the MPTP-treated primate, it has only been reliably documented in vervet monkeys (Guehl et al. 2003), and rodent models do not have this phenotype. In addition to an incomplete range of motor deficits, there are a variety of cognitive deficits also associated with PD that either are not demonstrated by these models or have yet to be evaluated. A few studies have looked at memory function in primates (Ferro et al. 2005; Ridley et al. 2006), but few have looked at depression and sleep dysfunction, which are common features of the disease. Recently, the MPTP-treated primate has been characterised for the development of drug-induced psychosis. In the long-term care of PD patients, the onset of psychosis is a major concern and is most commonly associated with the dopaminergic drug usage. Visanji et al. (2006) assessed agitation, obsessive grooming, stereotypies and hallucinatory-like responses to non-apparent stimuli, distinguishing these behaviours from dyskinesia. They suggest that the pharmacology of these behaviours reflects that observed in the clinic and suggests that the atypical neuroleptics, unlike haloperidol, were able to reduce these Ldopa-mediated behaviours without interfering with the antiparkinsonian action (Visanji et al. 2006).

\section{Validity of animal models of dyskinesia}

Although it was first made available in the 1960s, L-dopa remains the most effective symptomatic relief for PD sufferers. The beneficial effects of L-dopa in the clinic are generally well reflected in both rodent and non-human primate models of the disease. However, whilst it is the gold standard in terms of functional improvement afforded by a pharmacotherapeutic, long-term treatment is not without significant problems involving wearing off, on-off fluctuations and dyskinetic side effects. To optimise L-dopa therapy and to develop drugs that circumvent these problems, it is necessary that these can also be reproduced in disease models.

Perhaps the greatest single factor compromising longterm L-dopa administration is the development of abnormal involuntary movements including dystonia and chorea, collectively known as dyskinesia. Within 5 years of starting Ldopa treatment, $40 \%$ of patients will develop dyskinesia, by 10 years this figure reaches $90 \%$ and can be a major limiting factor in the progression of dopaminergic therapy (Ahlskog and Muenter 2001). L-dopa-induced dyskinesia (LID) show different temporal patterns, and can be produced at the peak effect of L-dopa (so-called 'peak-dose dyskinesia') or can also be present at the onset and decline of each L-dopa dose ('end-of-dose dyskinesia'). In addition, L-dopa therapy does not provide a continuous, smooth improvement in motor function but can produce abrupt motor fluctuations in an unpredictable 'on/off' fashion and a premature 'wearing off' of L-dopa-mediated symptomatic relief. As with the evaluation of symptomatic benefit, the determination of side effect profiles, in particular dyskinesiogenic potential, have commonly been assessed in the toxin-based models of PD. Initially, the predominant model used was the MPTP-treated non-human primate. After chronic treatment with L-dopa, MPTP-treated monkeys developed choreic and dystonic movements that can be closely paralleled with the peakdose dyskinesia in patients. Indeed, LID in monkeys can be scored using clinical rating scales after only modest adaptation (Imbert et al. 2000).

The history of evaluating the side effect profile of L-dopa and newer drugs in the 6-OHDA-lesioned rat is more convoluted. Early reports suggested that the enhancement in amplitude of the contralateral rotational response was that of the basal ganglia sensitisation thought to underlie the development of dyskinesia (Papa et al. 1994; Mura et al. 2002). The analysis of dyskinesia in this model has now been developed, and study of the rotational behaviour is now supplemented by the analysis of fore and hindlimbs, jaw and torso movements that can be paralleled with dyskinesia in patients. These behaviours, which develop over time with repeated L-dopa administration, were first characterised in rats in 1998, by Cenci et al. and subsequently in mice (Lundblad et al. 2005) and have since been 
developed to increase the sensitivity and to include more behavioural characteristics (Winkler et al. 1999; SteeceCollier et al. 2003). Their temporal profile reflects that of peak-dose dyskinesia and movements involve both dystonic distortion of the torso and hindlimbs with hyperkinetic and occasionally dystonic movements of the forelimb and orolingual muscles.

A disadvantage in terms of experimental significance, but with greater parallels to the clinic, is the variability in the type of movements that are displayed by both L-dopatreated parkinsonian primates and rodents. Some may show a predominantly dystonic phenotype whilst others have greater choreoathetoid characteristics. Furthermore, the propensity to develop dyskinesia is variable; in the same way that some patients do not develop these side effects for many years, similarly a proportion of both primates and rodents show little dyskinesia even after extended administration of L-dopa.

Both of these models of dyskinesia have been extensively validated pharmacologically. In the clinic, apomorphine is capable of inducing short-lived dyskinesia; conversely dopamine agonists have less propensity to induce dyskinetic behaviours. Concordant with these clinical reports, the rodent and primate models of dyskinesia follows a similar profile of dyskinetic tendencies. Despite a lack of dyskinesia induction by chronic de novo administration of dopamine agonists, the administration of dopamine agonists to 6-OHDA lesioned rats or MPTP-treated primates that have developed dyskinesia after chronic L-dopa administration will elicit greater levels of abnormal movements. These models are further validated with other anti-dyskinetic therapies such as treatment with $D_{1}$, $D_{2}$ and $D_{3}$ antagonists (Monville et al. 2005), that have also been shown to exhibit a degree of clinical efficacy, again suggesting a reasonable degree of concordance between the clinical setting and pre-clinical models.

Although these behaviours convincingly resemble peak dose dyskinesia, the representation of other aspects of long term L-dopa administration is dependent on the model used. In the MPTP-treated primate, both 'end-of dose deterioration,' the 'on-off' phenomena and rebound worsening have been reported (Clarke et al. 1987, 1988; Kuoppamaki et al. 2002). In contrast, in the 6-OHDAlesion model, few of these facets of long-term L-dopa treatment occur. The L-dopa-induced rotational response has been reported to decrease in duration with repeated administration, which may reflect the wearing off that patients experience (Papa et al. 1994). However, this has not been consistently demonstrated (Lane et al. 2006; Lindgren et al. 2007) and is unconvincing as a reliable model of 'wearing off.' Furthermore, the sporadic and unpredictable failure of doses of L-dopa to promote motor behaviour in rodents had been thought to relate to the on/off fluctuations and drug resistant periods that are an increasing problem with chronic treatment. This has since been proven to be an artefact of intraperitoneal administration, such that subcutaneous application of the drug appears to eliminate the occurrence of these so-called 'dose failures'(Lindgren et al. 2007).

As discussed above, the models of dyskinesia have been validated using currently available compounds. However, as with the development of symptomatic therapies, the high face validity of these models does not appear to be translating into high predictive validity in the clinic. Idazoxan, yohimbine and JP-1370, all selective adrenergic $\alpha_{2 \mathrm{~A}}$ antagonists demonstrated good anti-dyskinetic potential in the MPTP-treated primate model of dyskinesia; however, clinical findings have been mixed, the results confounded by low tolerability. Nevertheless, it should be considered that assessment of yohimbine in the 6-OHDA lesioned rat predicted reduced dyskinesia but only at a dose that also reduced L-dopa efficacy on motor symptoms (Dekundy et al. 2007). In MPTP-treated non-human primates adenosine A2a antagonists promisingly produced more natural improvements in locomotor responding compared to L-dopa without inducing dyskinesia, yet in the clinical trials L-dopa induced dyskinesia were unchanged (Bara-Jimenez et al. 2003). The $5-\mathrm{HT}_{1 \mathrm{~A}}$ agonist sarizotan significantly reduced dyskinesia in both rodent and primate models of dyskinesia (Bibbiani et al. 2001). Again, despite positive results in initial trials (Olanow et al. 2004; Bara-Jimenez et al. 2005), a recent larger scale phase IIB clinical trial saw no benefit in the concomitant use of sarizotan with L-dopa on the presence or severity of dyskinesia (Goetz et al. 2007). Proponents of this compound suggest that the failure may be caused by the dopamine $\mathrm{D}_{2}$ receptor antagonism properties also possessed by sarozitan, but that does not explain the contrasting success observed in pre-clinical assessments. There may be other reasons related to the design of the trial and the continued administration of ongoing therapy that could provide explanations for these disappointing results, but the continued predictive validity of the model remains an open question. As highlighted by Fox et al. (2006), the accurate translation of animal model data into the clinic requires that the animal model is used appropriately and that the clinical trial is designed to test the resulting hypothesis (Fox et al. 2006). In the case of evaluating treatments for L-dopa induced dyskinesia, they suggest that the means of inducing dyskinesia and of assessing dyskinesia severity need to be consistent between the animal model and clinical environment (Fox et al. 2006). These two parameters can be very varied between clinical studies as well as between clinical and pre-clinical studies and the true predictive validity of the dyskinesia models will only be apparent when there is concordance between them. 


\section{Novel animal models of parkinsonism}

To represent PD more closely, alternative animal models have recently been described that at first glance may look more appropriate than the older, more established models. However, there has been a considerable amount of controversy surrounding them, and extensive refinement and validation is necessary to support these as first line models in PD therapeutics. The concepts for these models derive from recent developments in knowledge and revised hypotheses on the potential causes of PD. Thus, some of the genes involved in familial PD have associations with the ubiquitinating processes that direct proteins to be degraded by the proteasome (McNaught et al. 2001). This association, alongside the implied fault in protein processing that underlies Lewy body formation led to the determination of deficits in proteasome function in postmortem PD tissue (McNaught and Jenner 2001; McNaught et al. 2003). The first report of proteasome inhibition in rodents using the synthetic proteasome inhibitor (PSI) excitingly reported a pathology striking similar to PD; neuronal degeneration of both dopaminergic and nondopaminergic pathways, the presence of Lewy body-like inclusions and motor deficits were all described (McNaught et al. 2004). However, there has been considerable debate about the reproducibility of this technique as whilst some groups have had findings consistent with McNaught et al. (Schapira et al. 2006; Zeng et al. 2006) other similarly expert teams have been unable to discern any significant behavioural or morphological changes that can be directly ascribed to the administration of PSI (Bove et al. 2006; Kordower et al. 2006; Manning-Bog et al. 2006). This has cast doubt on the model and its potential in both primates and rodents and further work has been proposed by McNaught et al. (2006) to determine the cause of these discrepancies.

In a mechanism similar to pathological action of MPTP, pesticides associated with increased incidence of PD (rotenone and paraquat) act as inhibitors of the complex I component of the mitochondrial respiratory chain. Furthermore, in post-mortem tissue of patients with sporadic PD there is reduced activity of the same complex suggesting that complex I deficits are implicit in the disease (Schapira et al. 1990; Kingsbury et al. 2001; Mandel et al. 2005). This has led to the use of rotenone and paraquat in rodents as an alternate model of the disease (Uversky 2004). Extensive loss of striatal dopaminergic innervation is observed with repeat or long-term exposure to rotenone, and importantly, there is an accumulation of $\alpha$-synuclein into aggregates (Betarbet et al. 2002). The extent and lesion size and type is highly variable in this model leading to a need for very high numbers of animals to be used in each study. Other difficulties include different sensitivities to rotenone between rat strains have led to difficulties with survival, this difference in vulnerability may be an asset to the model, as although it complicates experimental work, it does bear similarities with the variability of PD (Hoglinger et al. 2006). The concordance with PD has been questioned by the determination that there is extensive extra-nigral cell death that is not consistent with the pathology of $\mathrm{PD}$, for example striatal neurones appear more vulnerable and are more consistently degenerated than nigral neurons (Ferrante et al. 1997; Hoglinger et al. 2003; Lapointe et al. 2004; Zhu et al. 2004). In addition, the accumulation of tau protein may be more abundant than $\alpha$ synuclein (Hoglinger et al. 2005). Despite some pathological similarities, the validity of the model for therapeutic purposes has yet to be shown. Whilst L-dopa is highly effective at inducing improvements in motor function in the rotenone model (Alam and Schmidt 2004), other drugs have not been tested. Both of the proteasome inhibition and rotenone models have the potential to be considerably more representative of PD than those currently in widespread use. The non-dopaminergic denervation and presence of protein accumulations in addition to extensive nigrostriatal degeneration is considerable progress in the development of experimental models of the disease. However, in order for them to become standard models of PD, their consistency and validity must be convincingly demonstrated.

The development of animal models allows us to screen and refine potential therapeutic strategies for their clinical efficacy. However, whilst the animal models have been validated based on the available treatments, it is not necessarily implicit that drugs developed on the basis of these animal models will work in the clinic. Whilst the motor deficits commensurate with $\mathrm{PD}$ have been well modelled in the 6-OHDA-lesioned rat and MPTP-treated primates and existing anti-parkinsonian drugs appear to validate the models, new pharmacotherapeutics designed to improve symptoms of the disease are not translating well into the clinic. Additionally, although there appears to be a higher level of model-to-clinic concordance when looking at anti-dyskinetic treatment strategies, there are still significant discrepancies. It must be acknowledged that the translation of potential therapies for PD from preclinical experimentation into clinical trials is a two-way process. The responsibility lies with the pre-clinical scientists to use the available models in a consistent way, so as to provide the most meaningful and comparative data that can be interpreted in the clinic, but similarly the design of clinical trials should reflect the evidence provided by successful pre-clinical experiments in animal models. The concordance of animal models with the human disease has, thus far, facilitated the development of a range of therapies for PD. However, the continued development of novel treatment approaches depends on the correct translation from the bench to the patient and ongoing model development based on the findings of clinical trials. 
Acknowledgements The authors would like to thank the UK Parkinson's Disease Society for their financial support of Dr Emma Lane.

\section{References}

Ahlskog JE, Muenter MD (2001) Frequency of levodopa-related dyskinesias and motor fluctuations as estimated from the cumulative literature. Mov Disord 16(3):448-458

Alam M, Schmidt WJ (2004) L-DOPA reverses the hypokinetic behaviour and rigidity in rotenone-treated rats. Behav Brain Res 153(2):439-446

Alexander GM, Schwartzman RJ, Brainard L, Gordon SW, Grothusen JR (1992) Changes in brain catecholamines and dopamine uptake sites at different stages of MPTP parkinsonism in monkeys. Brain Res 588(2):261-269

Bara-Jimenez W, Sherzai A, Dimitrova T, Favit A, Bibbiani F, Gillespie M, Morris MJ, Mouradian MM, Chase TN (2003) Adenosine $\mathrm{A}(2 \mathrm{~A})$ receptor antagonist treatment of Parkinson's disease. Neurology 61(3):293-296

Bara-Jimenez W, Dimitrova T, Sherzai A, Favit A, Mouradian MM, Chase TN (2004) Effect of monoamine reuptake inhibitor NS 2330 in advanced Parkinson's disease. Mov Disord 19(10):1183-1186

Bara-Jimenez W, Bibbiani F, Morris MJ, Dimitrova T, Sherzai A, Mouradian MM, Chase TN (2005) Effects of serotonin 5-HT1A agonist in advanced Parkinson's disease. Mov Disord 20(8): 932-936

Baronti F, Mouradian MM, Conant KE, Giuffra M, Brughitta G, Chase TN (1992) Partial dopamine agonist therapy of levodopainduced dyskinesias. Neurology 42(6):1241-1243

Baumgarten HG, Klemm HP, Lachenmayer L, Bjorklund A, Lovenberg W, Schlossberger HG (1978) Mode and mechanism of action of neurotoxic indoleamines: a review and a progress report. Ann N Y Acad Sci 305:3-24

Bernheimer H, Birkmayer W, Hornykiewicz O, Jellinger K, Seitelberger F (1973) Brain dopamine and the syndromes of Parkinson and Huntington. Clinical, morphological and neurochemical correlations. J Neurol Sci 20(4):415-455

Betarbet R, Sherer TB, Greenamyre JT (2002) Animal models of Parkinson's disease. Bioessays 24(4):308-318

Bibbiani F, Oh JD, Chase TN (2001) Serotonin 5-HT1A agonist improves motor complications in rodent and primate parkinsonian models. Neurology 57(10):1829-1834

Bove J, Zhou C, Jackson-Lewis V, Taylor J, Chu Y, Rideout HJ, Wu DC, Kordower JH, Petrucelli L, Przedborski S (2006) Proteasome inhibition and Parkinson's disease modeling. Ann Neurol 60(2):260-264

Braak H, Del Tredici K, Bratzke H, Hamm-Clement J, Sandmann-Keil D, Rub U (2002) Staging of the intracerebral inclusion body pathology associated with idiopathic Parkinson's disease (preclinical and clinical stages). J Neurol 249(Suppl 3):III/1-5

Breese GR, Baumeister AA, McCown TJ, Emerick SG, Frye GD, Crotty K, Mueller RA (1984) Behavioral differences between neonatal and adult 6-hydroxydopamine-treated rats to dopamine agonists: relevance to neurological symptoms in clinical syndromes with reduced brain dopamine. J Pharmacol Exp Ther 231 (2):343-354

Carlsson A, Lindqvist M, Magnusson T (1957) 3,4-Dihydroxyphenylalanine and 5-hydroxytryptophan as reserpine antagonists. Nature 180(4596): 1200

Clarke CE, Sambrook MA, Mitchell IJ, Crossman AR (1987) Levodopa-induced dyskinesia and response fluctuations in primates rendered parkinsonian with 1-methyl-4-phenyl-1,2,3,6tetrahydropyridine (MPTP). J Neurol Sci 78(3):273-280
Clarke CE, Boyce S, Sambrook MA, Stahl SM, Crossman AR (1988) Behavioural effects of (-)-4-propyl-9-hydroxynaphthoxazine in primates rendered parkinsonian with 1-methyl-4-phenyl-1,2,3,6tetrahydropyridine. Naunyn Schmiedebergs Arch Pharmacol 338 (1):35-38

Dekundy A, Lundblad M, Danysz W, Cenci MA (2007) Modulation of L-DOPA-induced abnormal involuntary movements by clinically tested compounds: further validation of the rat dyskinesia model. Behav Brain Res 179(1):76-89

Donnan GA, Kaczmarczyk SJ, Solopotias T, Rowe P, Kalnins RM, Vajda FJ, Mendelsohn FA (1986) The neurochemical and clinical effects of 1-methyl-4-phenyl-1,2,3,6-tetrahydropyridine in small animals. Clin Exp Neurol 22:155-164

Dowd E, Monville C, Torres EM, Dunnett SB (2005) The Corridor Task: a simple test of lateralised response selection sensitive to unilateral dopamine deafferentation and graft-derived dopamine replacement in the striatum. Brain Res Bull 68(1-2):24-30

Eidelberg E, Brooks BA, Morgan WW, Walden JG, Kokemoor RH (1986) Variability and functional recovery in the $N$-methyl-4phenyl-1,2,3,6-tetrahydropyridine model of parkinsonism in monkeys. Neuroscience 18(4):817-822

Fahn S, Libsch LR, Cutler RW (1971) Monoamines in the human neostriatum: topographic distribution in normals and in Parkinson's disease and their role in akinesia, rigidity, chorea, and tremor. J Neurol Sci 14(4):427-455

Feany MB, Bender WW (2000) A Drosophila model of Parkinson's disease. Nature 404(6776):394-398

Ferrante RJ, Schulz JB, Kowall NW, Beal MF (1997) Systemic administration of rotenone produces selective damage in the striatum and globus pallidus, but not in the substantia nigra. Brain Res 753(1):157-162

Ferro MM, Bellissimo MI, Anselmo-Franci JA, Angellucci ME, Canteras NS, Da Cunha C (2005) Comparison of bilaterally 6OHDA- and MPTP-lesioned rats as models of the early phase of Parkinson's disease: histological, neurochemical, motor and memory alterations. J Neurosci Methods 148(1):78-87

Fleming SM, Fernagut PO, Chesselet MF (2005) Genetic mouse models of parkinsonism: strengths and limitations. NeuroRx 2 (3):495-503

Forno LS, Langston JW, DeLanney LE, Irwin I, Ricaurte GA (1986) Locus ceruleus lesions and eosinophilic inclusions in MPTPtreated monkeys. Ann Neurol 20(4):449-455

Fox SH, Lang AE, Brotchie JM (2006) Translation of nondopaminergic treatments for levodopa-induced dyskinesia from MPTPlesioned nonhuman primates to phase IIa clinical studies: keys to success and roads to failure. Mov Disord 21(10):1578-1594

Frackiewicz EJ, Jhee SS, Shiovitz TM, Webster J, Topham C, Dockens RC, Whigan D, Salazar DE, Cutler NR (2002) Brasofensine treatment for Parkinson's disease in combination with levodopa/carbidopa. Ann Pharmacother 36(2):225-230

Goetz CG, Damier P, Hicking C, Laska E, Muller T, Olanow CW, Rascol O, Russ H (2007) Sarizotan as a treatment for dyskinesias in Parkinson's disease: a double-blind placebo-controlled trial. Mov Disord 22(2):179-186

Grondin R, Goulet M, Di Paolo T, Bedard PJ (1996) Cabergoline, a long-acting dopamine D2-like receptor agonist, produces a sustained antiparkinsonian effect with transient dyskinesias in parkinsonian drug-naive primates. Brain Res 735(2):298-306

Guehl D, Pessiglione M, Francois C, Yelnik J, Hirsch EC, Feger J, Tremblay L (2003) Tremor-related activity of neurons in the 'motor' thalamus: changes in firing rate and pattern in the MPTP vervet model of parkinsonism. Eur J Neurosci 17(11):2388-2400

Hansard MJ, Smith LA, Jackson MJ, Cheetham SC, Jenner P (2004) The monoamine reuptake inhibitor BTS 74398 fails to evoke established dyskinesia but does not synergise with levodopa in MPTP-treated primates. Mov Disord 19(1):15-21 
Hoglinger GU, Feger J, Prigent A, Michel PP, Parain K, Champy P, Ruberg M, Oertel WH, Hirsch EC (2003) Chronic systemic complex I inhibition induces a hypokinetic multisystem degeneration in rats. J Neurochem 84(3):491-502

Hoglinger GU, Lannuzel A, Khondiker ME, Michel PP, Duyckaerts C, Feger J, Champy P, Prigent A, Medja F, Lombes A, Oertel WH, Ruberg M, Hirsch EC (2005) The mitochondrial complex I inhibitor rotenone triggers a cerebral tauopathy. J Neurochem 95 (4):930-939

Hoglinger GU, Oertel WH, Hirsch EC (2006) The rotenone model of parkinsonism - the five years inspection. J Neural Transm Suppl (70):269-272

Imbert C, Bezard E, Guitraud S, Boraud T, Gross CE (2000) Comparison of eight clinical rating scales used for the assessment of MPTP-induced parkinsonism in the Macaque monkey. J Neurosci Methods 96(1):71-76

Iravani MM, Syed E, Jackson MJ, Johnston LC, Smith LA, Jenner P (2005) A modified MPTP treatment regime produces reproducible partial nigrostriatal lesions in common marmosets. Eur J Neurosci 21(4):841-854

Iwamoto ET, Loh HH, Way EL (1976) Circling behavior in rats with 6-hydroxydopamine or electrolytic nigral lesions. Eur J Pharmacol 37(2):339-356

Jackson-Lewis V, Przedborski S (2007) Protocol for the MPTP mouse model of Parkinson's disease. Nat Protoc 2(1):141-151

Jenner P, Rupniak NM, Rose S, Kelly E, Kilpatrick G, Lees A, Marsden CD (1984) 1-Methyl-4-phenyl-1,2,3,6-tetrahydropyridineinduced parkinsonism in the common marmoset. Neurosci Lett 50 $(1-3): 85-90$

Kingsbury AE, Cooper M, Schapira AH, Foster OJ (2001) Metabolic enzyme expression in dopaminergic neurons in Parkinson's disease: an in situ hybridization study. Ann Neurol 50(2):142-149

Kirik D, Rosenblad C, Bjorklund A (1998) Characterization of behavioral and neurodegenerative changes following partial lesions of the nigrostriatal dopamine system induced by intrastriatal 6-hydroxydopamine in the rat. Exp Neurol 152(2):259-277

Kordower JH, Kanaan NM, Chu Y, Suresh Babu R, Stansell J 3rd, Terpstra BT, Sortwell CE, Steece-Collier K, Collier TJ (2006) Failure of proteasome inhibitor administration to provide a model of Parkinson's disease in rats and monkeys. Ann Neurol 60 (2):264-268

Kowall NW, Hantraye P, Brouillet E, Beal MF, McKee AC, Ferrante RJ (2000) MPTP induces alpha-synuclein aggregation in the substantia nigra of baboons. Neuroreport 11(1):211-213

Kuoppamaki M, Al-Barghouthy G, Jackson M, Smith L, Zeng BY, Quinn N, Jenner P (2002) Beginning-of-dose and rebound worsening in MPTP-treated common marmosets treated with levodopa. Mov Disord 17(6):1312-1317

Lane EL, Cheetham SC, Jenner P (2005) Repeated administration of the monoamine reuptake inhibitor BTS 74398 induces ipsilateral circling in the 6-hydroxydopamine lesioned rat without sensitizing motor behaviours. Eur J Neurosci 21(1):179-186

Lane EL, Cheetham SC, Jenner P (2006) Does contraversive circling in the 6-OHDA-lesioned rat indicate an ability to induce motor complications as well as therapeutic effects in Parkinson's disease? Exp Neurol 197(2):284-290

Langston JW, Forno LS, Rebert CS, Irwin I (1984) Selective nigral toxicity after systemic administration of 1-methyl-4-phenyl1,2,5,6-tetrahydropyrine (MPTP) in the squirrel monkey. Brain Res 292(2):390-394

Lapointe N, St-Hilaire M, Martinoli MG, Blanchet J, Gould P, Rouillard C, Cicchetti F (2004) Rotenone induces non-specific central nervous system and systemic toxicity. Faseb J 18(6): $717-719$

Lewy FH (1914) Zur pathologischen anatomie der Paralysis Agitans. Dtsch. Z. Nervenheilk 1:50-55
Linazasoro G (2004) Recent failures of new potential symptomatic treatments for Parkinson's disease: causes and solutions. Mov Disord 19(7):743-754

Lindgren HS, Rylander D, Ohlin KE, Lundblad M, Cenci MA (2007) The "motor complication syndrome" in rats with 6-OHDA lesions treated chronically with L-DOPA: relation to dose and route of administration. Behav Brain Res 177(1):150-159

Ljungberg T, Ungerstedt U (1976) Reinstatement of eating by dopamine agonists in aphagic dopamine denervated rats. Physiol Behav 16(3):277-283

Lundblad M, Usiello A, Carta M, Hakansson K, Fisone G, Cenci MA (2005) Pharmacological validation of a mouse model of LDOPA-induced dyskinesia. Exp Neurol 194(1):66-75

Maeda T, Kannari K, Shen H, Arai A, Tomiyama M, Matsunaga M, Suda T (2003) Rapid induction of serotonergic hyperinnervation in the adult rat striatum with extensive dopaminergic denervation. Neurosci Lett 343(1):17-20

Mandel S, Grunblatt E, Riederer P, Amariglio N, Jacob-Hirsch J, Rechavi G, Youdim MB (2005) Gene expression profiling of sporadic Parkinson's disease substantia nigra pars compacta reveals impairment of ubiquitin-proteasome subunits, SKP1A, aldehyde dehydrogenase, and chaperone HSC-70. Ann N Y Acad Sci 1053:356-375

Manning-Bog AB, Reaney SH, Chou VP, Johnston LC, McCormack AL, Johnston J, Langston JW, Di Monte DA (2006) Lack of nigrostriatal pathology in a rat model of proteasome inhibition. Ann Neurol 60(2):256-260

Maratos EC, Jackson MJ, Pearce RK, Jenner P (2001) Antiparkinsonian activity and dyskinesia risk of ropinirole and L-DOPA combination therapy in drug naive MPTP-lesioned common marmosets (Callithrix jacchus). Mov Disord 16(4):631-641

Marshall JF, Levitan D, Stricker EM (1976) Activation-induced restoration of sensorimotor functions in rats with dopaminedepleting brain lesions. J Comp Physiol Psychol 90(6):536-546

McNaught KS, Jenner P (2001) Proteasomal function is impaired in substantia nigra in Parkinson's disease. Neurosci Lett 297 (3):191-194

McNaught KS, Olanow CW, Halliwell B, Isacson O, Jenner P (2001) Failure of the ubiquitin-proteasome system in Parkinson's disease. Nat Rev Neurosci 2(8):589-594

McNaught KS, Belizaire R, Isacson O, Jenner P, Olanow CW (2003) Altered proteasomal function in sporadic Parkinson's disease. Exp Neurol 179(1):38-46

McNaught KS, Perl DP, Brownell AL, Olanow CW (2004) Systemic exposure to proteasome inhibitors causes a progressive model of Parkinson's disease. Ann Neurol 56(1):149-162

McNaught KS, Olanow CW (2006) Proteasome inhibitor-induced model of Parkinson's disease. Ann Neurol 60:243-247

Meissner W, Prunier C, Guilloteau D, Chalon S, Gross CE, Bezard E (2003) Time-course of nigrostriatal degeneration in a progressive MPTP-lesioned macaque model of Parkinson's disease. Mol Neurobiol 28(3):209-218

Monville C, Torres EM, Dunnett SB (2005) Validation of the 1-dopainduced dyskinesia in the 6-OHDA model and evaluation of the effects of selective dopamine receptor agonists and antagonists. Brain Res Bull 68(1-2):16-23

Mura A, Mintz M, Feldon J (2002) Behavioral and anatomical effects of long-term L-dihydroxyphenylalanine (L-DOPA) administration in rats with unilateral lesions of the nigrostriatal system. Exp Neurol 177(1):252-264

Nicklas WJ, Vyas I, Heikkila RE (1985) Inhibition of NADH-linked oxidation in brain mitochondria by 1-methyl-4-phenyl-pyridine, a metabolite of the neurotoxin, 1-methyl-4-phenyl-1,2,5,6-tetrahydropyridine. Life Sci 36(26):2503-2508

Olanow CW, Damier P, Goetz CG, Mueller T, Nutt J, Rascol O, Serbanescu A, Deckers F, Russ H (2004) Multicenter, open-label, trial of sarizotan in Parkinson disease patients with levodopa- 
induced dyskinesias (the SPLENDID Study). Clin Neuropharmacol 27(2):58-62

Olsson M, Nikkhah G, Bentlage C, Bjorklund A (1995) Forelimb akinesia in the rat Parkinson model: differential effects of dopamine agonists and nigral transplants as assessed by a new stepping test. J Neurosci 15(5 Pt 2):3863-3875

Papa SM, Engber TM, Kask AM, Chase TN (1994) Motor fluctuations in levodopa treated parkinsonian rats: relation to lesion extent and treatment duration. Brain Res 662(1-2):69-74

Park J, Kim SY, Cha GH, Lee SB, Kim S, Chung J (2005) Drosophila DJ-1 mutants show oxidative stress-sensitive locomotive dysfunction. Gene 361:133-139

Pifl C, Schingnitz G, Hornykiewicz O (1991) Effect of 1-methyl-4-phenyl1,2,3,6-tetrahydropyridine on the regional distribution of brain monoamines in the rhesus monkey. Neuroscience 44(3):591-605

Reavill C, Jenner P, Marsden CD (1983) Differentiation of dopamine agonists using drug-induced rotation in rats with unilateral or bilateral 6-hydroxydopamine destruction of ascending dopamine pathways. Biochem Pharmacol 32(5):865-870

Ridley RM, Cummings RM, Leow-Dyke A, Baker HF (2006) Neglect of memory after dopaminergic lesions in monkeys. Behav Brain Res 166(2):253-262

Schapira AH, Mann VM, Cooper JM, Dexter D, Daniel SE, Jenner P, Clark JB, Marsden CD (1990) Anatomic and disease specificity of NADH CoQ1 reductase (complex I) deficiency in Parkinson's disease. J Neurochem 55(6):2142-2145

Schapira AH, Cleeter MW, Muddle JR, Workman JM, Cooper JM, King RH (2006) Proteasomal inhibition causes loss of nigral tyrosine hydroxylase neurons. Ann Neurol 60(2):253-255

Schwarting RK, Huston JP (1996) The unilateral 6-hydroxydopamine lesion model in behavioral brain research. Analysis of functional deficits, recovery and treatments. Prog Neurobiol 50(2-3): 275-331

Smith LA, Gordin A, Jenner P, Marsden CD (1997) Entacapone enhances levodopa-induced reversal of motor disability in MPTP-treated common marmosets. Mov Disord 12(6):935-945

Steece-Collier K, Collier TJ, Danielson PD, Kurlan R, Yurek DM, Sladek JR Jr (2003) Embryonic mesencephalic grafts increase levodopa-induced forelimb hyperkinesia in parkinsonian rats. Mov Disord 18(12):1442-1454
Ungerstedt U (1971a) Adipsia and aphagia after 6-hydroxydopamine induced degeneration of the nigro-striatal dopamine system. Acta Physiol Scand Suppl 367:95-122

Ungerstedt U (1971b) Postsynaptic supersensitivity after 6-hydroxydopamine induced degeneration of the nigro-striatal dopamine system. Acta Physiol Scand Suppl 367:69-93

Uversky VN (2004) Neurotoxicant-induced animal models of Parkinson's disease: understanding the role of rotenone, maneb and paraquat in neurodegeneration. Cell Tissue Res 318(1):225-241

Visanji NP, Gomez-Ramirez J, Johnston TH, Pires D, Voon V, Brotchie JM, Fox SH (2006) Pharmacological characterization of psychosis-like behavior in the MPTP-lesioned nonhuman primate model of Parkinson's disease. Mov Disord 21(11): 1879-1891

Whishaw IQ, Woodward NC, Miklyaeva E, Pellis SM (1997) Analysis of limb use by control rats and unilateral DA-depleted rats in the Montoya staircase test: movements, impairments and compensatory strategies. Behav Brain Res 89(1-2):167-177

Winkler C, Bentlage C, Nikkhah G, Samii M, Bjorklund A (1999) Intranigral transplants of GABA-rich striatal tissue induce behavioral recovery in the rat Parkinson model and promote the effects obtained by intrastriatal dopaminergic transplants. Exp Neurol 155(2):165-186

Zeng BY, Bukhatwa S, Hikima A, Rose S, Jenner P (2006) Reproducible nigral cell death after systemic proteasomal inhibitor administration to rats. Ann Neurol 60(2):248-252

Zhou FC, Bledsoe S, Murphy J (1991) Serotonergic sprouting is induced by dopamine-lesion in substantia nigra of adult rat brain. Brain Res 556(1):108-116

Zhu C, Vourc'h P, Fernagut PO, Fleming SM, Lacan S, Dicarlo CD, Seaman RL, Chesselet MF (2004) Variable effects of chronic subcutaneous administration of rotenone on striatal histology. J Comp Neurol 478(4):418-426

Zigmond MJ, Stricker EM (1972) Deficits in feeding behavior after intraventricular injection of 6-hydroxydopamine in rats. Science 177(55):1211-1214

Zigmond MJ, Stricker EM (1973) Recovery of feeding and drinking by rats after intraventricular 6-hydroxydopamine or lateral hypothalamic lesions. Science 182(113):717-720 